\title{
Comprehensive Care
}

National Cancer Institute

\section{Source}

National Cancer Institute. Comprehensive Care. NCI Thesaurus. Code C52640.

Planned, non-emergency care that includes prevention and maintance and restoration of health. 